\title{
Predicting the Potential Market for Electric Vehicles
}

Jensen, Anders Fjendbo; Cherchi, Elisabetta; Mabit, Stefan Lindhard; Ortúzar, Juan de Dios

Published in:

Transportation Science

Link to article, DOI:

$10.1287 /$ trsc.2015.0659

Publication date:

2017

Document Version

Peer reviewed version

Link back to DTU Orbit

Citation (APA):

Jensen, A. F., Cherchi, E., Mabit, S. L., \& Ortúzar, J. D. D. (2017). Predicting the Potential Market for Electric Vehicles. Transportation Science, 51(2), 427-440. https://doi.org/10.1287/trsc.2015.0659

\section{General rights}

Copyright and moral rights for the publications made accessible in the public portal are retained by the authors and/or other copyright owners and it is a condition of accessing publications that users recognise and abide by the legal requirements associated with these rights.

- Users may download and print one copy of any publication from the public portal for the purpose of private study or research.

- You may not further distribute the material or use it for any profit-making activity or commercial gain

- You may freely distribute the URL identifying the publication in the public portal

If you believe that this document breaches copyright please contact us providing details, and we will remove access to the work immediately and investigate your claim. 
TS-2014-0228 Resubmission

\title{
PREDICTING THE POTENTIAL MARKET FOR ELECTRIC VEHICLES
}

\author{
Anders F. Jensen, Elisabetta Cherchi, Stefan L. Mabit, \\ Department of Transport, Technical University of Denmark, \\ Bygningstorvet 116, Building 116 Vest, 2800 Kgs. Lyngby, Denmark \\ e-mails: afjje@transport.dtu.dk; elich@transport.dtu.dk; slm@transport.dtu.dk \\ Juan de Dios Ortúzar, \\ Department of Transport Engineering and Logistics, \\ Centre for Urban Sustainable Development (CEDEUS), \\ Pontificia Universidad Católica de Chile, \\ Vicuña Mackenna 4860, Macul, Santiago 7820436, Chile \\ e-mail: jos@ing.puc.cl
}

\section{Abstract}

Forecasting the potential demand for electric vehicles is a challenging task. As most studies for new technologies rely on stated preference (SP) data, market share predictions will reflect shares in the SP data and not in the real market. Moreover, typical disaggregate demand models are suitable to forecast demand in relatively stable markets, but show limitations in the case of innovations. When predicting the market for new products it is crucial to account for the role played by innovation and how it penetrates the new market over time through a diffusion process. However, typical diffusion models in marketing research use fairly simple demand models. In this paper we discuss the problem of predicting market shares for new products and suggest a method that combines advanced choice models with a diffusion model to take into account that new products often need time to gain a significant market share. We have the advantage of a relatively unique databank where respondents were submitted to the same stated choice experiment before and after experiencing an electric vehicle. Results show that typical choice models forecast a demand that is too restrictive in the long period. Accounting for the diffusion effect, instead allows predicting the slow penetration of the initial years and a faster market share increase after diffusion takes place.

Keywords: electric vehicles, forecasting; diffusion, discrete choice 


\section{INTRODUCTION}

Electric vehicles (EV) have faced extreme difficulties in developing into a mass marketed product. With recent introductions of new models from several producers, the need for reliable predictions of future demand is greater than ever. Discrete choice modelling is a popular tool for predicting demand. A well-known problem related to the prediction of EV demand, is connected to the fact that most discrete choice models for new technologies rely on hypothetical stated preference (SP) data. When these models are used for prediction, alternative-specific constants (ASCs) and eventually scale, need to be adjusted to reflect the fact that unobserved factors may vary between the hypothetical setting and the real market (Cherchi \& Ortúzar, 2006; Train, 2009).

Consequently, many studies on demand for EV using SP data only present model estimations and/or trade-offs between coefficients but do not provide forecasts (Achtnicht 2012; Beggs et al. 1981; Daziano \& Bolduc 2013; Hidrue et al. 2011; Ito et al. 2013; Potoglou \& Kanaroglou 2007; Ramjerdi \& Rand 2000). A few studies (Adler et al. 2003; Knockaert 2005) present models estimated on SP data which are intended for larger simulations systems, but do not report how these models would be integrated. Aware of the limitations of SP data, most studies use the estimated coefficients to compare EV market shares in different scenarios (Calfee 1985; Bunch et al. 1993; Ewing \& Sarigöllü 2000; Dagsvik et al. 2002; Mabit \& Fosgerau 2011; Glerum et al. 2013; Hackbarth \& Madlener 2013), but do not claim that these are actual forecasts. Brownstone et al. (2000) used joint RP/SP models finding that the joint RP/SP estimation gave a much lower EV market share (18\% instead of $42 \%)$ than when using only SP data. Batley et al. (2004) used SP data collected in the UK and re-calibrated the alternative specific constants (ASC) using the USA market shares found by Brownstone et al. (2000). Using results from other countries to re-estimate the ASC might be an appropriate solution in the absence of better information, but results are sensitive to the reference market share used to recalibrate the ASC, so care must be taken on the type of market considered as reference and its characteristics. Moreover, Jensen et al. (2013) show that direct experience on EV changes individual 
preferences for specific EV characteristics. Thus, this effect needs to be considered also when forecasting the potential demand for EV.

Typical disaggregate demand models (as used in all papers above) are suitable to forecast the demand for relatively stable markets, but show limitations in the case of innovation. Glerum et al. (2013) tried to explicitly account for the lack of EV market shares but they did not deal with the evolution of demand over time. There is an important literature on dynamic vehicle holding models (where the holding in one time period depends on the past or future time periods) and vehicle transaction models, but this mainly refers to conventional cars and does not explicitly consider the effect of innovation. Innovations often diffuse slowly and need time to obtain a significant market share; unfortunately, this process cannot be properly reproduced with typical demand models. The diffusion process has been defined as that by which an innovation is communicated through certain channels over time among members of a social system (Rogers, 2010). The behavioural assumption in these models is that an innovation is first adopted by a segment called innovators, who are willing to adopt a product despite, perhaps, a high price or low reliability; in contrast, adoption by another segment, the imitators, depends on the number of adoptions that have already occurred.

We envisage that the diffusion effect can be one of the reasons behind the delay in EV market penetration. Although the characteristics of EV and their recharging options play a crucial role in explaining their potential demand, the effect of diffusion needs to be considered when predicting market shares. Marketing studies include many examples of investigations related with diffusion processes, but these mainly look at a single product and if they account for substitution among products they typically use very simple demand models.

When forecasting new technologies, growth curves are widely used to describe the slow product growth in the introduction period, followed by a rapid exponential growth period when barriers to product adoption fall and when, finally, the product reaches a market share limit. Among the most popular are the models by Bass, (1969) and Gompertz (1825). Since the original version of these 
models does not account for the influence of competitive products, several extensions have been suggested in the literature. For example, Weerahandi \& Dalal (1992) and Jun \& Kim (2011) combine a Bass diffusion model with a logit model to simultaneously capture the diffusion process and the replacement process in a multi-product framework. However, they use aggregate demand models based on time series market shares data to estimate the joint diffusion/substitution model. Weerahandi \& Dalal (1992) used a disaggregate demand model, but they only included two variables at the disaggregate level.

For the plug-in hybrid electric vehicle market, Eppstein et al. (2011) formulated an agent based vehicle consumer choice model taking into account the fact that a potential customer's purchase decision is affected by media coverage and social interactions. However, as they did not have sufficient data to make quantitative predictions, the model was used to explore potential nonlinear interactions between the various influences on the market. Higgins et al. (2012) developed a diffusion model incorporating multi-criteria analysis and choice models to estimate the adoption of electric vehicles. Their study focused especially on the geographical uptake of electric vehicles in a region and the effect of different policy incentives.

More recently, Kieckhäfer et al. (2014) set up a simulation system specifically for alternative fuel cars where a richer disaggregate demand model (estimated separately in previous research) was integrated into a dynamic simulation system to allow modelling the important feedbacks in demand due to the dynamic evolution of EV and their recharging characteristics. However, they do not account for the typical diffusion effect of Bass-type models. Previous papers (i.e. Shepherd et al. 2012; Struben \& Sterman 2008) have presented similar simulation systems integrating disaggregate demand models and system dynamic models where the diffusion effect (i.e. reference rate of social exposure) is included. However, in these studies, the parameters in the dynamic part of the models are exogenously defined rather than estimated. Moreover, the disaggregate models estimated with SP data are adjusted to the real market exogenously from the diffusion process and reflect the same problems associated with forecasting using SP data. In fact, Shepherd et al. (2012) initially scaled the EV shares estimated with 
SP data by a factor of $6 / 20$, which is the ratio between the market shares estimated with the RP/SP and the SP-only specification found by Brownstone et al. (2000). Based on these shares they computed fairly high ASC, which were adjusted to reproduce suggested UK market forecasts.

The objective of this paper is to discuss the prediction of EV market shares and to suggest a method that combines a diffusion model, as typically estimated in the marketing literature, with advanced discrete choice models, where all the parameters of the joint substitution/diffusion model are estimated jointly and the disaggregate model estimated with SP data is adjusted to the real market endogenously in the diffusion process. We believe our proposal is an improvement over all previous approaches.

The rest of the paper is organized as follows. In section 2 we discuss the problem of predicting market shares. We describe first the method using discrete choice models and then the diffusion model. In Section 3 we discuss the problem of forecasting the market shares for EV and present a method to combine the diffusion models with advanced discrete choice models. In section 4 we give an example of how the methodology can be applied and describe the data needed for this. Section 5 presents the results of the application and discusses our most interesting findings. In section 6 we summarize and conclude.

\section{PREDICTING DEMAND FOR NEW PRODUCTS}

\subsection{Predicting with disaggregate choice models}

Demand models are estimated mainly to forecast demand. They are estimated for a specific context and then, assuming that the demand curve is stable (i.e. the coefficients do not vary), they are used to forecast demand in future years. Demand models are typically estimated at the disaggregate level, while forecasts usually represent the behaviour of an entire population (or market segment). At the same time, disaggregate models are often estimated using a sample of observations from the population. Then, given a disaggregate model estimated on a sample, to use this model in forecasting usually requires: (1) to aggregate the model to the population segment of interest; (2) to change the value of the attributes according to the policy scenarios and to represent the variation in decision 
makers' characteristics, and (3) to adjust the segments dimension to reflect changes over time in the number of decision makers in the population.

Different methods can be used to aggregate models estimated at the disaggregate level (see Ben-Akiva \& Lerman 1985, pages 131-148; Ortúzar \& Willumsen 2011, pages 338-341; Train 2009, pages 2932). The easiest way is to use average values and if the model is linear this is correct. But as discrete choice models are non-linear, aggregation based on a representative consumer is biased. Therefore aggregate values for the market shares are most often obtained with sample enumeration. Purposely designed synthetic samples can also be used in an enumeration approach (Gunn et al. 1982). With this method, a random sample of the population is used as representative of the entire population. If the sample is non-random, the population is classified into groups, and the estimated market share is calculated as a weighed sum of the within-class forecasts.

Consider for simplicity a logit model estimated on a sample assumed to be representative of the population: $\hat{\beta}$ is a column vector of estimated coefficients, $x_{n i}$ a vector of attributes, $\lambda$ the unknown scale of the model in the estimation context (usually set equal to one and not estimated), $A \hat{S} C$ a full set of estimated alternative specific constants ensuring that the model reproduces the observed market shares in the sample and $N$ the reference population in the estimation year. Then the aggregate market share of alternative $i$ is given by:

$$
M S_{i}=\frac{1}{N} \sum_{n=1}^{N} P_{i}=\frac{1}{N} \sum_{n=1}^{N} \frac{\exp \left(\lambda\left(A \hat{S} C_{i}+\hat{\beta} \cdot x_{n i}\right)\right)}{\sum_{j} \exp \left(\lambda\left(A \hat{S} C_{j}+\hat{\beta} \cdot x_{n j}\right)\right)}
$$

The model can then be used to forecast demand based on assumptions about the values that the variables entering the model will have in this scenario. Often it is necessary to adjust the ASC and the scale to reflect the fact that unobserved factors are different between the estimation and prediction contexts. This is the typical problem of model transferability (Ortúzar \& Willumsen 2011, pages 341345). It is also the typical problem encountered when models are estimated using SP data. The 
problem can be easily solved if aggregate real market shares are available for the new context. If the scale also needs calibration, market shares for two points in time are necessary.

Once a model (correctly adjusted to reproduce the baseline market shares) is available, an iterative method can be used to adjust the constants. This consists in comparing the estimated and actual shares until they are sufficiently close to each other (Ortúzar \& Willumsen, 2001, page 235):

$A \hat{S} C_{j}^{t=1}=A \hat{S} C_{j}^{t=0}+\ln \left(M S_{j} / \widehat{M S}_{J}^{t=0}\right)$

This simple method allows for adjusting the ASC only. To adjust both for ASC and scale we would need to update the vector of attributes $\left(x_{n j}^{t}\right)$ at the future time $t$ and re-estimate the model using the following utility:

$U_{n j}^{t}=A S C_{j}^{t}+\lambda^{t}\left(\hat{\beta} \cdot x_{n j}^{t}\right)+\varepsilon_{n j}^{t}$

where $\beta$ are assumed to be fixed over time and equal to the coefficients estimated in the base year, while the coefficients $\left(A S C_{j}^{t}, \lambda^{t}\right)$ are estimated for the new situation.

Unfortunately, very often SP data is used to study products not currently available, for which there is not yet a real market share available. This represents one of the major problems when dealing with prediction of the EV market. If the coefficients are estimated using RP and SP data jointly (as in Brownstone et al., 2000), then the scale of the model is correct because it is set to the RP data, while the constants can be adjusted according to the RP situation. If the coefficients are estimated using only SP data, there is no clear solution (Cherchi \& Ortúzar 2006). For example, Glerum et al. (2013) made some corrections of their SP model to reflect the real market situation. Since part of their sample only had the choice between a conventional Renault and an electric Renault, they imputed an extra alternative that represented other gasoline cars on the market and corrected the ASC so that the ratios between Renault and the competitors were preserved. However, for the EV alternative, they assumed 
that the response rates to the SP questionnaire would represent the state of the market if electric vehicles were released at the time of the study. They found an EV market share of $27 \%$, which is quite high for the initial year.

\subsection{Diffusion models}

Many different growth curve models have been used to describe the diffusion of new technologies. The Gompertz and Bass models are among the most popular ones, built on the behavioural theory that an innovative product is usually adopted first by a few people ("innovators"), who in turn, influence others ("imitators") to adopt it. Both models follow an S-shape to describe the diffusion of a product. The Gompertz curve model is specified by the equation:

$$
Y_{t}=M \cdot e^{-a \cdot e^{b t}}
$$

where $Y_{t}$ is the cumulative number of adoptions at time $t, M$ is the number of potential adopters, and $a$ and $b$ are parameters to be estimated by fitting them to the observed number of cumulative adoptions.

The diffusion model due to Bass (1969) states that the number of new adopters at period $t$ is defined as:

$a_{t}=\left(p+q \frac{Y_{t-1}}{M}\right)\left(M-Y_{t-1}\right)$

where $Y_{t-1}$ is the cumulative number of adoptions that occurred before period $t, p$ is the coefficient of innovation, capturing intrinsic tendency to adopt as well as the effect of time invariant external influences, and $q$ is the coefficient of imitation, capturing the fact that the adoption probability of customers increases with the proportion of eventual adopters who have already adopted.

An important characteristic of the Bass model is that it is symmetric around the inflection point whereas this point occurs earlier in the growth trend for the Gompertz model. Moreover, in the Bass model the interpretation of the coefficients $p$ and $q$ can be directly associated with innovators and 
imitators, while in the Gomperz model the coefficients $a$ and $b$ do not have this direct interpretation. All the researches that have tried to combine diffusion and substitution effects have been based on the Bass model.

The Bass model is a good starting point for forecasting the long-term penetration pattern of new technologies and products under two types of conditions (Lilien et al. 2000): (1) a product has recently been introduced and the penetration has been observed for a few time periods, or (2) the product has not yet been introduced, but it is similar in some way to existing products or technologies with a known diffusion history. These conditions are required to estimate the unknown coefficients in the Bass model. According to Lilien et al. (2000), data must be available for at least four periods to allow estimation of $p$ and $q$. If no such data is available, parameters estimated for historical innovations that are similar to the innovation being studied are often used instead. Moreover, to make use of equation (5), RP data describing whether the customers adopted the product or not are needed.

Several approaches have been suggested to extend the basic diffusion model to account for the interrelationship among various products (i.e. the substitution dynamic effects among products). In particular, Jun \& Park (1999) present one of the first attempts to incorporate diffusion and substitution effects (i.e. a discrete choice model) into an integrated model. More specifically, they include the diffusion effect (though different from the Bass specification) directly into the utility of subscribing for the durable technology $k$ (i.e. of generation $k$ ) at time period $t$ :

$V_{t}^{(i, k)}=q^{(i, k)}\left(t-\tau^{k}+1\right)+\beta^{(i, k)} \cdot x_{t}^{(i, k)}$,

where $x_{t}^{(i, k)}$ is a vector of attribute values for generation $k$ at time period $t, \beta^{(i, k)}$ is a vector of corresponding coefficients, $q^{(i, k)}$ is the time dependent diffusion effect related to each product generation and $\tau^{k}$ is the year when a product of generation $k$ was introduced into the market. The superscript $(i, k)$ refers to the general case of an individual who owns a technology of generation $i$ and subscribes for a technology of generation $k$, with $k>i$. Jun \& Park (1999) argue that the time variables 
may capture most of the effects of the unavailable attributes (or exogenous variables). Note that they do not include ASC in the specification, which they do not comment on further. This is interesting because, although they do not mention it, in one application of their model they estimated diffusion terms specific for each generation, which - to some extent - captures the same effect as the ASC in a typical discrete choice model.

Assuming an identically and independently distributed (iid) Extreme Value type 1 (EV1) error term for first time buyers at time $t$, Jun \& Park (1999) computed the probability $\left(P_{t}^{0, k}\right)$ to purchase or not and which generation $k$ to purchase; in the case of consumers already using $i$-th generation products at time $t$, they computed the probability ( $P_{t}^{i, k}$ ) to upgrade or not and which generation $k$ to choose:

$$
P_{t}^{0, k}=\frac{\exp \left(V_{t}^{0, k}\right)}{\exp (c)+\sum_{j} \exp \left(V_{t}^{0, j}\right)} ; \quad P_{t}^{i, k}=\frac{\exp \left(V_{t}^{i, k}\right)}{\sum_{j} \exp \left(V_{t}^{i, j}\right)} ; \quad \forall(j, k)>i,
$$

where $c$ is a constant, included in the utility of the non-purchase alternative, assumed to remain constant in the diffusion period.

If the number of products in use for each time period (such as the total number of car registrations) is available and hence data are available for both new purchases and up-graders from one category to subsequent categories, then the net sales are the sum of the first buyer plus the new upgraders minus the current adopters of generation $k$ who change to another generation $j$ :

$$
\begin{gathered}
S_{t}^{k}=\left(M_{t}-Y_{t-1}\right) \cdot P_{t}^{0, k}+\sum_{i=1}^{k-1} Y_{t-1}^{i} P_{t}^{i, k}-Y_{t-1}^{k} \sum_{j=k+1}^{n_{t}} P_{t}^{k, j}, \\
k=i+1, i+2, \ldots, n_{t}
\end{gathered}
$$

where $M_{t}$ is the potential market share at time $t, Y_{t-1}$ the total number of products in use at time $t-1$ (this accounts implicitly for the replacement by newer generations; as such, the total number of products in a generation can decrease while cumulative sales do not decrease and is therefore different 
from the cumulative sales), $Y_{t-1}^{i}$ is the number of adopters using products of the $i$-th generation at time $t-1$, and $n_{t}$ is the number of generations available at time $t$.

If the data does not allow distinguishing between replacement and first purchase demand (i.e. total sales in each time period), they use a similar method where first time buyers and replacements are aggregated. In this case, the number of sales in each period would be:

$$
S_{t}^{k}=\left(M_{t}-Y_{t-1}\right) \cdot P_{t}^{k}=\left(M_{t}-Y_{t-1}\right) \cdot \frac{\exp \left(V_{t}^{k}\right)}{\exp (c)+\sum_{j} \exp \left(V_{t}^{j}\right)}
$$

with $V_{t}^{k}=q^{k}\left(t-\tau^{k}+1\right)+\beta^{k} \cdot x_{t}^{k}$.The cumulative sales for year $t$ would then be:

$Y_{t}=Y_{t-1}+\sum_{k=1}^{n_{t}} S_{t}^{k}$

Although the model is fairly general, for estimation purposes they made the assumption that the potential market share at each time period $t$ was equal to the potential market share of each generation $k$ (i.e. $M_{t}=M_{k}$ ) and it was not affected by the marketing variables, but remained constant. In their most advanced application, the utility (7) depends only on one attribute (the price), but its coefficient is different for the various generations of the product; the final model includes a total of nine coefficients estimated simultaneously (four price coefficients, four market share values and the constant $c$ ).

Further developments of the joint diffusion/substitution model have mainly worked in the direction of accounting for the different substitution effects that can take place over time (such as the possibility that new adopters can switch back to the old product or postpone the choice decision skipping previous generations and directly adopting a newer generation, (e.g. Jun \& Kim 2011; Jiang \& Jain 2012) and to link them better with the diffusion model (such as including the marketing mix variables in the utility specification, (e.g. Jun et al. 2002; Jun \& Kim 2011). But the substitution models are 
always used with basic structures. As opposed to Jun \& Park (1999), most of them model explicitly the Bass formulation in equation (5).

In these more advanced diffusion/substitution models all coefficients are estimated simultaneously. This is complicated and requires much data on the real market shares. This is (at least so far) not available for the EV market. On the other hand the substitution models used in the diffusion literature are very simple; they include few attributes to define the product (usually only the cost) and they are specified at the aggregate level. Since EVs are still under development, almost all of their characteristics are likely to change significantly in the future. Therefore, it is crucial to use a substitution model that captures properly the effect of these characteristics on EV demand.

\section{COMBINING DISAGGREGATE MODELS AND DIFFUSION MODELS}

As mentioned before, transport research uses fairly sophisticated disaggregated substitution models, but diffusion is not accounted for. Instead, in marketing, diffusion is accounted for in a highly sophisticated way but the substitution models are fairly simple. The idea behind the method we propose here consists in replacing the probabilities in (8) with models estimated at a disaggregate level, assuming that the trade-offs are stable over time and re-estimate only the scale and ASC to adjust to the observed market shares. Hence, following Jun \& Park's work (though the method can be applied to any type of diffusion model), the probability of choosing category $k$ can be written as:

$$
\begin{gathered}
P(0, k)=\frac{\exp \left(\lambda\left(A S C_{t}^{(0, k)}+q^{(0, k)}\left(t-\tau^{(0, k)}+1\right)+\hat{\beta}^{(0, k)} \cdot x_{t}^{k}\right)\right)}{\exp \left(A S C_{\text {nobuy }}\right)+\sum_{j} \exp \left(\lambda\left(A S C_{t}^{(0, j)}+q^{(0, j)}(0, k)+\hat{\beta}^{(0, j)} \cdot x_{t}^{j}\right)\right)} \\
P(i, k)=\frac{\exp \left(\lambda\left(A S C_{t}^{(i, k)}+q^{(i, k)}\left(t-\tau^{(i, k)}+1\right)+\hat{\beta}^{(i, k)} \cdot x_{t}^{k}\right)\right)}{\sum_{j} \exp \left(\lambda\left(A S C_{t}^{(i, j)}+q^{(i, j)}\left(t-\tau^{(i, j)}+1\right)+\hat{\beta}^{(i, j)} \cdot x_{t}^{j}\right)\right)},(k, j)>i
\end{gathered}
$$

where $\hat{\beta}$ is a vector of estimated coefficients from the disaggregate model. The remaining coefficients, in particular ASC and scale, are estimated in the joint diffusion/substitution model. The model structure can be any discrete choice model less restrictive than the simple multinomial logit (MNL) 
model. For example, Brownstone et al. (2000) and Hensher \& Greene (2001) applied mixed logit (ML) models to study vehicle type choice. Then, when estimating demand using equations (8) or (9), instead of estimating the $\beta^{\prime} s$, as in Jun \& Park (1999), we need to estimate the scale $(\lambda)$ and ASC together with the diffusion, while the $\beta^{\prime} s$ are estimated from a disaggregate model.

The demand model in equation (11) can be extended to account for different types of substitution as in the more advanced diffusion models briefly discussed in section 2.2. However, if a suitable disaggregate model is available, the procedure can be applied without further assumptions. Therefore, the application of the joint diffusion/discrete choice model to predict EV demand is mainly limited by the lack of sufficient data with enough detail (as all transactions are needed) and with enough variability to allow estimating the coefficients of the diffusion model. It is also important to mention that choice of an EV is part of the car transaction problem and, as such, is very complicated. However we can assume that individuals choose first whether to buy a car and then whether to adopt and hence, eventually, choose an EV. In this paper we focus on the second part, while the first can be modelled as described by de Jong (1996) or Goldberg (1998).

\section{DATA AND MODELS USED TO PREDICT EV MARKET SHARES}

In this section we show how the model in equations (8) and (11) can be applied to the specific case of predicting the market penetration of EV. The practical application of the model is of course limited by the data available and the model structures estimated with them. Note that all the data used in this case pertains to fully battery propelled $\mathrm{EV}$, and hence the application is only applicable to this specific type of EV. Also, given the type of data available, we do not distinguish between replacement and first purchase demand and we do not include a non-purchase alternative. The section also provides a brief description of our data, the disaggregate model available, and the scenarios set up to make forecasts. 


\subsection{Empirical model development}

Following the specification from Jun \& Park (1999), we define the utilities of choosing an EV and a conventional internal combustion engine car (ICVs) as follows:

$V_{t}^{E V}=A S C^{E V}+q^{E V}\left(t-\tau^{E V}+1\right)+\hat{\beta}^{E V} \cdot x_{t}^{E V}$

$V_{t}^{I C V}=A S C^{I C V}+\hat{\beta}^{I C V} \cdot x_{t}^{I C V}$

where $q^{E V}$ is the parameter catching the effect of diffusion, $\tau^{E V}$ is the time when the EV alternative is introduced in the market, $\hat{\beta}^{E V}$ and $\hat{\beta}^{I C V}$ are the coefficients for $x_{t}^{E V}$ and $x_{t}^{I C V}$, which are characteristics of the EV and ICV alternatives, respectively, and ASC are the alternative specific constants (at least one needs to be normalised to zero). The diffusion effect is only included in the utility for the EV alternative, as we assume that the ICV alternative has been fully adopted by the population.

Let $M_{E V}$ be the market potential of the EV alternative and $Y_{t-1}^{E V}$ be the cumulative number of EV sales before time period $t$. The number of EV sales in period $t$ can be then calculated as:

$S_{t}^{E V}=\left(M^{E V}-Y_{t-1}^{E V}\right) \cdot \operatorname{Pr}\left(E V_{t}\right)$

Or more specifically as:

$S_{t}^{E V}=\left(M^{E V}-Y_{t-1}^{E V}\right) \cdot \frac{\exp \left[A S C^{E V}+q^{E V} \cdot\left(t-\tau^{E V}+1\right)+\lambda\left(\hat{\beta}^{E V} \cdot x_{t}^{E V}\right)\right]}{\exp \left[A S C^{I C V}+\lambda\left(\hat{\beta}^{I C V} \cdot x_{t}^{I C V}\right)\right]+\exp \left[A S C^{E V}+q^{E V}\left(t-\tau^{E V}+1\right)+\lambda\left(\hat{\beta}^{E V} \cdot x_{t}^{E V}\right)\right]}$

where $\hat{\beta}^{E V}$ and $\hat{\beta}^{I C V}$ are estimated in the disaggregate model using SP data (as described below) and get fixed in the diffusion process. $q_{E V}, \lambda$ and the ASC are estimated as part of the diffusion process. Note that since the ASC and $q$ are estimated, only the coefficients estimated in the disaggregate choice model needs to be explicitly scaled by $\lambda$. For simplicity we assumed that all demand is absorbed in the market, which is not always the case (Keith 2012). If supply constraints exist this could bias the diffusion model parameters. 


\subsection{Disaggregate demand model for substitution}

To model the substitution demand between EV and gasoline cars, we used an error components ML model estimated on the basis of stated choice (SC) data including the choice between an EV and an ICV. The probability that individual $n$ chooses alternative $k$ is given by:

$P_{n}(k)=\frac{\exp \left(A S C^{k}+\hat{\beta}^{k} \cdot x_{n}^{k}\right)}{\sum_{\mathrm{j} \in \mathrm{C}_{\mathrm{n}}} \exp \left(A S C^{j}+\hat{\beta}^{j} \cdot x_{n}^{j}\right)}$,

where $x_{n}^{j}$ is a vector of vehicle attributes (including charging options for EVs), $\hat{\beta}^{j}$ is a vector of coefficients associated with the above variables and $A S C_{j}$ are the typical alternative-specific constants. $C_{n}$ is the choice set available to each individual $n$.

Data was available from a survey performed in Denmark in 2012 and 2013. Respondents answering the survey had participated in a demonstration project where they could use an EV (it was provided to the household) for a period of three months. To measure any change in preferences caused by the experience with the EV, the same SC experiment was conducted before and after this period. The attributes used in the SC experiment included purchase price, driving distance, driving costs (represented by fuel or electricity costs), driving performance (defined as top speed), environmental performance (defined as carbon emissions), charging options and battery lifetime for the EV. A detailed description of the survey can be found in Jensen et al. (2014).

For the purpose of this paper we used the parameters estimated jointly with both data collected before and after the direct experience with EV. From the model estimation we found that individual preferences indeed changed for some attributes after having tried the $\mathrm{EV}$, and it is interesting to test this effect in forecasting. Details on the models estimated can be found in Jensen \& Cherchi (2014). We believe that, based on more experience about how the EV actually fits into the household, respondents' choices should be more likely to represent an actual decision process. Table 1 reports the values of the estimated coefficients used in this paper. 
Table 1: Estimated parameters of the discrete choice model

\begin{tabular}{|c|c|c|c|c|c|}
\hline & \multirow[b]{2}{*}{ Unit } & \multicolumn{2}{|c|}{ Before } & \multicolumn{2}{|c|}{ After } \\
\hline & & Value & $\begin{array}{c}\text { Robust } \\
\text { t-test }\end{array}$ & Value & $\begin{array}{c}\text { Robust } \\
\text { t-test }\end{array}$ \\
\hline $\operatorname{ASC}(\alpha), E V$ & & 0.258 & 0.24 & -4.440 & -3.41 \\
\hline Standard deviation for panel effect $(\sigma), \mathrm{EV}$ & & 1.630 & 8.08 & 1.950 & 7.55 \\
\hline Purchase price (PP) & {$[100,000 \mathrm{DKK}]$} & -1.750 & -9.80 & -1.750 & -9.80 \\
\hline Propulsion costs, EV & {$[D K K / \mathrm{km}]$} & -6.200 & -5.06 & -5.410 & -3.41 \\
\hline Propulsion costs, ICV & {$[D K K / \mathrm{km}]$} & -1.760 & -3.63 & -1.180 & -1.97 \\
\hline Driving range, $\mathrm{EV}$ & {$[\mathrm{km}]$} & 1.060 & 3.01 & 2.790 & 5.27 \\
\hline Driving range, ICV & {$[\mathrm{km}]$} & 0.149 & 2.26 & 0.149 & 2.26 \\
\hline Carbon dioxide emissions & {$[\mathrm{g} / \mathrm{km}]$} & -0.035 & -0.25 & -0.035 & -0.25 \\
\hline Battery stations & [units] & 0.463 & 2.03 & 1.160 & 4.21 \\
\hline Public charging, slow, shop & [dummy] & 0.241 & 1.10 & 0.241 & 1.10 \\
\hline Public charging, slow, city & [dummy] & 0.037 & 0.15 & 0.651 & 2.32 \\
\hline Public charging, fast, shop & [dummy] & 0.569 & 2.52 & 0.569 & 2.52 \\
\hline Public charging, fast, city & [dummy] & 0.398 & 1.67 & 0.398 & 1.67 \\
\hline Scale & & & & 0.907 & 0.73 \\
\hline \multicolumn{2}{|l|}{ Number of observations } & \multicolumn{2}{|c|}{1322} & \multicolumn{2}{|c|}{1344} \\
\hline \multicolumn{2}{|l|}{ Number of individuals } & \multicolumn{2}{|c|}{290} & \multicolumn{2}{|c|}{290} \\
\hline \multicolumn{2}{|l|}{ Log likelihood } & \multicolumn{4}{|c|}{-1219} \\
\hline \multicolumn{2}{|l|}{ Null Log likelihood } & \multicolumn{4}{|c|}{-1848} \\
\hline \multicolumn{2}{|l|}{$\bar{\rho}^{2}$} & \multicolumn{4}{|c|}{0.325} \\
\hline
\end{tabular}

\subsection{Data on market shares}

As discussed in section 2.2, the coefficients of the diffusion model can be estimated on historical data for a similar product (if data for the new product is not available). Finding a similar innovation, however, can be challenging. Kurani et al. (1994) compared the introduction of EV into the car market with the introduction of microwave ovens into the oven market. In the beginning, consumers had concerns about taste and health, which caused a slow penetration. Eventually, however, consumers discovered that the microwave was not a replacement for the conventional oven but rather a supplement with some new useful options, and market penetration increased from 15\% in 1980 to 75 $80 \%$ by 1992 (Kerin et al. 2003). Both the EV and the microwave are "high learning products", in the sense that to fully use their qualities individuals have to make behavioural adjustments (Gärling \& Thøgersen 2001). We considered the possibility of using the microwave as a similar innovation. 
However, we questioned whether households would really consider the EV as a supplement to the conventional car in Denmark, where only $60 \%$ of households own a car and $76 \%$ of those who own a car only have this one car. Moreover, the latest generations of EV are much improved in terms of size, comfort and driving performance so that the attributes, with the exception of driving range, are now more comparable with those of a conventional car. A similar innovation could also be represented by earlier fuel innovations of the car such as hybrids, liquid gas or other ethanol-based cars. Lamberson (2008), for example, estimated the diffusion of hybrid electric vehicles and compared the performance of the Bass and Gompertz models. However, fully battery electric vehicles are quite different from hybrid electric vehicles, in terms of the behavioural adjustments required by users (due mainly to driving range, recharging time and so on).

In this paper we used information about revealed demand in Denmark. However we also collected data about EV market shares in Norway and the Netherlands, and performed several tests using these data from similar contexts. For all three countries, the number of registrations (i.e. stock data), were available on an annual basis: from 1993 to 2013 for Denmark and from 2008 to 2013 for the Netherlands and Norway. Furthermore, monthly new car registrations were available from 2008 to 2013 for Norway and from 2008 until August 2014 (inclusive) for Denmark. Norway is currently the country with the highest share of EV in Europe and is quickly reaching a share of total registrations of 1\%. The development in cumulative sales for Norway and Denmark is shown in Figure 1. Even though the total car market is actually larger in Denmark (182,000 new car registrations in 2013) compared to Norway (142,000 new car registration in 2013), the number of EV registrations is much larger in Norway compared to Denmark. It is important to remember that both Denmark and Norway have fairly high registration taxes on cars and in both countries battery electric vehicles are exempted from these taxes. However, in Norway, EV are also exempted from value added tax (VAT), which is $25 \%$ in both countries. Furthermore, Norwegian EV drivers also benefit from free charging at public charging points, free parking at public parking places, exemption from congestion charges and ferry charges, just as they are allowed to drive in bus lanes. The theoretical formulation suggested in this 
paper allows accounting for all these effects and for the effect of their possible removal/reduction in future years. However to do that, the coefficients for the relevant policies need to be estimated and included in the disaggregate demand model. The survey used to estimate the parameters for the demand model in our application, only provides the parameters to adjust for the monetary incentives, but this are not distinguished from the coefficient of the purchase price.

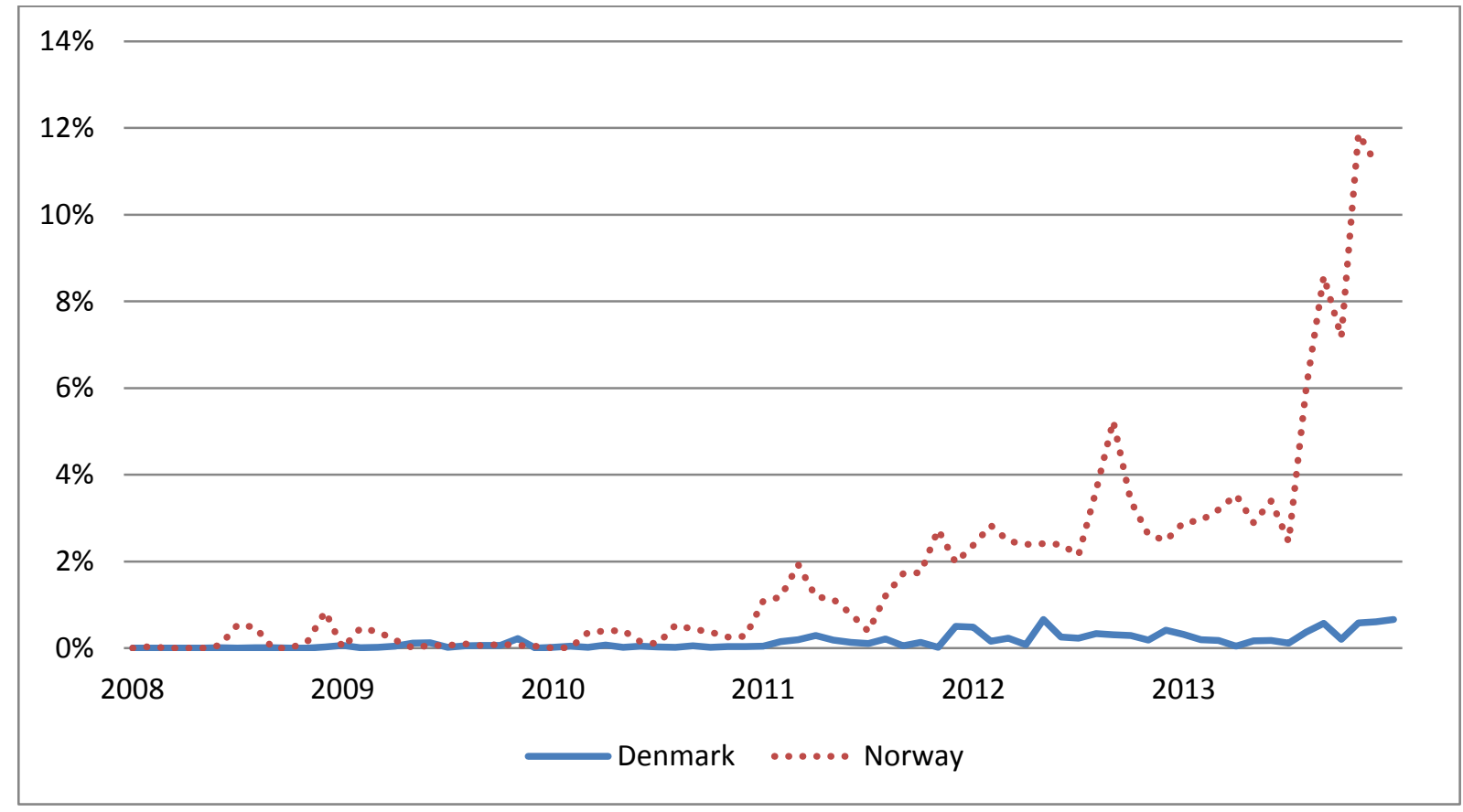

Data from Norway was available from Opplysningsrådet for Veitrafikken AS (Norwegian Information Committee for Road Traffic). Data from Denmark was available from the Danish Car Importers Association.

Figure 1: EV monthly share of car sales in Norway and Denmark from 2008 to 2014

\subsection{Scenarios for forecasting and simulation}

When making predictions it is necessary to make some assumptions about the development of the different factors included in the model. With the aim to show the effect of the assumptions made in forecasting (i.e. ASC and scale recalibration, and the effect of diffusion), we only set up simple scenarios where the EV experienced most improvements due to technological development. Forecasting the characteristics of EV is not an easy task and requires several assumptions about regulation beyond the car market (i.e. it depends on government's policy on petrol and energy and so on). Since the objective of this empirical application was not to forecast precisely the future market, 
but to test the method proposed, we built scenarios that have realistic values and that at the same time allowed for some non-marginal improvements in EV characteristics, in order to disentangle better the substitution effect from the diffusion effect. The attribute values assumed to forecast the choice model are shown in Table 2 (the attributes for the choice model forecast and the historical data are based on the following sources: European Centre for Mobility Documentation, Danish EV alliance, Norwegian Information Council for Road Traffic, Clever A/S). In the scenarios defined we assumed that there would be no major changes in current policy incentives in Denmark.

Table 2: Scenarios for the forecasting model from 2008 to 2020

\begin{tabular}{ccccccccccccccc}
\hline & \multicolumn{2}{c}{ Carbon } & \multicolumn{2}{c}{ Purchase price } & \multicolumn{2}{c}{ Fuel costs } & $\begin{array}{c}\text { Driving } \\
\text { range }\end{array}$ & $\begin{array}{c}\text { slow } \\
\text { city }\end{array}$ & $\begin{array}{c}\text { slow } \\
\text { shop }\end{array}$ & $\begin{array}{c}\text { fast } \\
\text { city }\end{array}$ & $\begin{array}{c}\text { fast } \\
\text { shop }\end{array}$ & $\begin{array}{c}\text { Battery } \\
\text { swap }\end{array}$ \\
\hline year & GAS & EV & GAS & EV & GAS & EV & GAS & EV & EV & EV & EV & EV & EV \\
\hline & {$[\mathrm{g} / \mathrm{km}]$} & {$[\mathrm{g} / \mathrm{km}]$} & {$[\mathrm{DKK}]$} & {$[\mathrm{DKK}]$} & $\begin{array}{c}{[\mathrm{DKK}} \\
/ \mathrm{km}]\end{array}$ & $\begin{array}{c}{[\mathrm{DKK}} \\
\mathrm{km}]\end{array}$ & {$[\mathrm{km}]$} & {$[\mathrm{km}]$} & {$[\%]$} & {$[\%]$} & {$[\%]$} & {$[\%]$} & {$[\mathrm{items}]$} \\
\hline $\mathbf{2 0 0 8}$ & 1.33 & 0.67 & 154,000 & 240,000 & 0.63 & 0.26 & 800 & 100 & 0.00 & 0.00 & 0.00 & 0.00 & 0.00 \\
\hline $\mathbf{2 0 0 9}$ & 1.29 & 0.72 & 154,000 & 240,000 & 0.62 & 0.26 & 800 & 150 & 0.01 & 0.01 & 0.00 & 0.00 & 0 \\
\hline $\mathbf{2 0 1 0}$ & 1.19 & 0.67 & 154,000 & 230,000 & 0.62 & 0.26 & 800 & 150 & 0.01 & 0.01 & 0.00 & 0.00 & 0 \\
\hline $\mathbf{2 0 1 1}$ & 1.19 & 0.56 & 154,000 & 230,000 & 0.61 & 0.26 & 800 & 150 & 0.01 & 0.02 & 0.00 & 0.01 & 5 \\
\hline $\mathbf{2 0 1 2}$ & 1.19 & 0.45 & 154,000 & 230,000 & 0.60 & 0.26 & 800 & 150 & 0.05 & 0.05 & 0.01 & 0.03 & 17 \\
\hline $\mathbf{2 0 1 3}$ & 1.19 & 0.43 & 154,000 & 230,000 & 0.60 & 0.25 & 800 & 150 & 0.06 & 0.06 & 0.04 & 0.04 & 0 \\
\hline $\mathbf{2 0 1 4}$ & 1.19 & 0.41 & 152,756 & 225,000 & 0.59 & 0.25 & 800 & 155 & 0.12 & 0.12 & 0.10 & 0.10 & 0 \\
\hline $\mathbf{2 0 1 5}$ & 1.19 & 0.38 & 152,110 & 222,500 & 0.58 & 0.25 & 800 & 160 & 0.17 & 0.17 & 0.16 & 0.16 & 0 \\
\hline $\mathbf{2 0 1 6}$ & 1.19 & 0.36 & 151,457 & 220,000 & 0.57 & 0.25 & 800 & 176 & 0.23 & 0.23 & 0.21 & 0.21 & 0 \\
\hline $\mathbf{2 0 1 7}$ & 1.18 & 0.33 & 150,796 & 217,500 & 0.56 & 0.24 & 800 & 192 & 0.28 & 0.28 & 0.27 & 0.27 & 0 \\
\hline $\mathbf{2 0 1 8}$ & 1.17 & 0.31 & 150,127 & 215,000 & 0.56 & 0.24 & 800 & 208 & 0.34 & 0.34 & 0.32 & 0.32 & 0 \\
\hline $\mathbf{2 0 1 9}$ & 1.15 & 0.29 & 149,451 & 211,950 & 0.55 & 0.24 & 800 & 208 & 0.39 & 0.39 & 0.38 & 0.38 & 0 \\
\hline $\mathbf{2 0 2 0}$ & 1.14 & 0.26 & 148,766 & 185,995 & 0.54 & 0.24 & 800 & 208 & 0.45 & 0.45 & 0.44 & 0.44 & 0 \\
\hline
\end{tabular}

As previously discussed, it is highly probable that at some point, as the EV market matures, there may be a reduction in policy incentives. However, we decided not to correct for this as we know very little about when and what would be changed and because the scope of this paper was on the methodology rather than providing exact forecasts of the future market for specific countries.

Our results allow seeing that, with our data and assumptions, the time horizon of 2020 is a long enough period to appreciate the curve of the typical diffusion process. However, a longer period would probably be needed to test and fully appreciate other important effects, such as incentives, supply strategies, or industrial plans. 
To model the time of the initial purchase, we used monthly sales data of EV in Denmark from January 2008 to August 2014 (both inclusive). The sales data are for private and company registrations of personal cars, but do not include cars registered for the demonstration project mentioned earlier (this included $195 \mathrm{EV}$, which is a large share of the EV car registrations so far). We currently have data available from January 2005, but this was before the first fully factory-made EVs were available on the Danish market, and in the period before 2008, only six EV were sold.

\section{RESULTS}

In this section we present and compare the results (i.e. prediction of EV demand) obtained by applying the methodologies described in section 2. In particular, we compare the following methods: (1) use DCM estimated in the base year (2013) with the SC data; (2) use the estimated DCM but recalibrating the ASC based on revealed aggregate sales data in Denmark 2013; (3) account for the effect of diffusion, assuming that the probability of adoption and the probability of choosing an EV are independent. The results for the first two methods are reported in section 5.1 and those for the last method in section 5.2.

\subsection{Predictions using only the discrete choice models}

In this first method we predict demand for the 2013 and 2020 scenarios simply using all the estimated parameters (including the ASC for the EV). As shown in Table 3, the market share increases from $25 \%$ to $66 \%$ due to the assumed improvements of the attributes (as reported in Table 2). As expected, the predicted market in 2013 is far above the revealed market share for the Danish market. This is in line with the findings of Brownstone et al. (2000), where the market share for EV were reduced when SP data was jointly estimated with RP data.

Table 3: EV Market share prediction of new car registrations

\begin{tabular}{lcccc}
\hline & \multicolumn{2}{c}{ Before experience } & \multicolumn{2}{c}{ After experience } \\
\hline Choice model only & $\mathbf{2 0 1 3}$ & $\mathbf{2 0 2 0}$ & $\mathbf{2 0 1 3}$ & $\mathbf{2 0 2 0}$ \\
\hline No calibration & 0.25 & 0.66 & 0.04 & 0.43 \\
Calibration & 0.003 & 0.02 & 0.003 & 0.06 \\
\hline
\end{tabular}


In the second method, we adjusted the ASC to reproduce the revealed market share in 2013, based on the new car registration data from Denmark. We adjusted the ASC in the model so that it simulated the market share of $0.3 \%$ in 2013. This method is quite restrictive. The EV market is kept at a very low level, and even with fairly large improvements in the attribute values, only a $2 \%$ market share is reached in 2020. Table 3 shows the same simulation based on the parameters estimated on the "after data" (i.e. after respondents have experience with EV). These parameters seem to give a more realistic picture of the current market share as the model now predicts a market share of $4 \%$ without calibration. However, the model still seems quite restrictive (not too different from the model estimated with the "before data") as only a $6 \%$ market share is forecasted for 2020 . These results are similar to those reported by Kieckhäfer et al. (2014) who showed very restrictive shares for future EV markets, despite the inclusion of system dynamics in their model, because the diffusion effect was not included as part of the dynamic system.

We are not dismissive of a 2-6\% EV market share in 2020, which could actually be a plausible result. However, we believe such increase is small given the major improvement assumed in the scenario and compared to the increase of $66 \%$ predicted by the un-calibrated model. As discussed, this effect is related to the fact that the ASC re-calibrated in the discrete choice models do not vary across the years. Then, if diffusion effects are captured by the ASC instead of being explicitly modelled (as in the diffusion/substitution model proposed in this paper), then we are not able to correctly measure the dynamic effect of the diffusion, but only its initial or final value, assuming erroneously that either the initial or the final condition will stay stable over time.

The examples above suggest that there are indeed time-dependent factors not included in the model. As discussed earlier, the ASC should represent all unobserved factors, but an important unobserved factor not considered is precisely the diffusion process, which was still at an early stage in 2013. 


\subsection{Predictions accounting for diffusion}

Assuming that the number of EV sales in a time period is equal to the number of EV adopters in that period, we first use the Bass model to explain the diffusion of EV in Denmark. Hence equation (5) becomes:

$S_{t}=\left(p+q \frac{Y_{t-1}}{M}\right)\left(M-Y_{t-1}\right)$

Estimating the Bass model on monthly sales data from Denmark between 2008 and 2013 (both inclusive), yielded the following parameters using non-linear least squares estimation:

Table 4: Parameter estimates of the Bass model

\begin{tabular}{lccc}
\hline & & \multicolumn{2}{c}{$95 \%$ confidence interval } \\
\cline { 3 - 4 } Parameter & Value & Min & Max \\
\hline $\boldsymbol{M}$ & 1189760 & $-4.01 \mathrm{E}+08$ & $4.03 \mathrm{E}+08$ \\
$\mathbf{p}$ & $2.22 \mathrm{E}-06$ & -0.00074 & 0.000748 \\
$\mathbf{q}$ & 0.0482 & 0.0228 & 0.0735 \\
\hline F value & & 73.90 & \\
Pr $>\mathrm{F}$ & & $<.0001$ & \\
\hline
\end{tabular}

Only the parameter for imitation is significant, but we did not expect otherwise. The parameter for innovators has been low and insignificant in all tests done. We also estimated the model using the data from the Netherlands and Norway, which currently has the highest EV share of car registrations in Europe, but did not find significant improvements compared to the Danish case. The insignificant parameter for $M$ means that we do not have sufficient data to estimate the overall market with precision yet, but the method can still be used to describe the evolution of the market to some defined overall market share. As discussed by Lilien et al. (2000), when using non-linear least squares estimation, the estimates of $M$ are often too low. Thus, it is often preferable to use exogenous data rather than having it as a part of the estimation procedure.

Based on these parameter estimates, a Bass model was used to illustrate the prediction based only on the diffusion model. We set $p$ to zero and for $M$ we assumed that the potential EV market was equal to 
half of the current car owning families, which are about 877,000 families. The size of the potential market is difficult to validate. As making a good assumption for $M$ is extremely difficult, we preferred to make a simple assumption for this study, as this fitted well with the objective of testing the methodology proposed ${ }^{1}$. To indicate the uncertainty of the model, we also calculated the number of sales for the minimum and maximum values of $q$; results are shown in Figure 2.

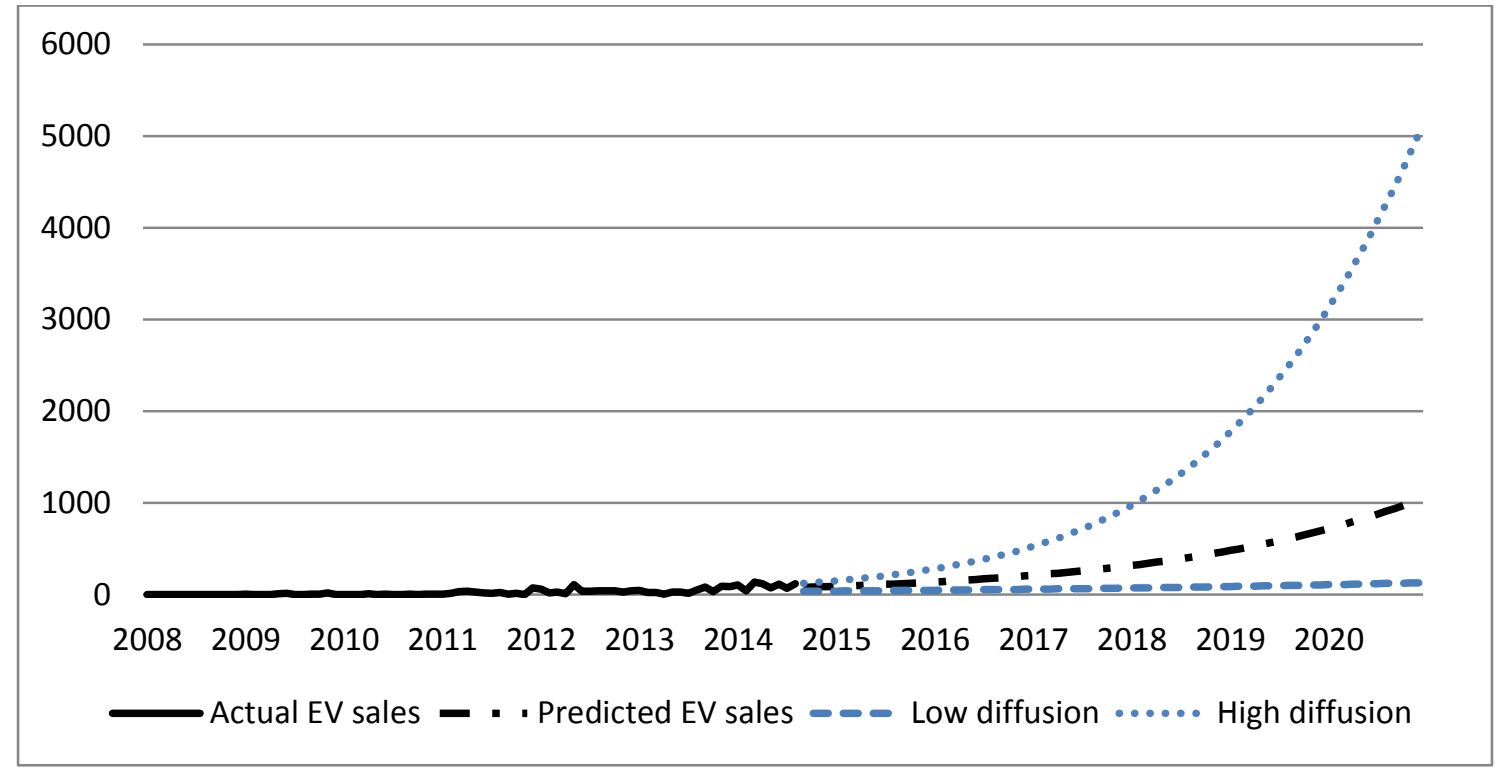

Figure 2: Monthly number of initial EV purchases

In the low diffusion scenario, only $130 \mathrm{EV}$ are sold per month in 2020, whereas in the high diffusion scenario around 5,000 EV are sold each month. The latter corresponds to approximately $30 \%$ of the new car sales of January 2014. Clearly, the uncertainty is very high, but as also indicated in Figure 2 the estimation is based on a very low number of actual EV sales from 2008 to 2013.

1 Several studies (Christensen et al. 2010; Greaves et al. 2014; Pearre et al. 2011) have used travel distance distributions to determine potential EV market sizes. They investigated the potential use of EV for everyday travel by household members based on data on internal combustion vehicles (ICV). This approach implicitly assumes that EV would be used exactly in the same way as ICV, which is a strong (not necessarily realistic) assumption. If the characteristics of EV improve (e.g. the purchase price becomes better than, or at least comparable with) that of ICV, people might be willing to change their travel behavior, implying different distances covered. 
The diffusion models as such are single product models and, hence, implicitly consider EV to be one single product that does not change over the years. In the following paragraph we combine a diffusion model with a choice model, to take into account that the characteristics of the EV and their competitors are not constant.

\subsection{Prediction accounting for choice and diffusion}

The joint diffusion and choice model presented in equation (11) was estimated using the same data as before. For the diffusion variable (i.e. number of months after market introduction), we found that a better fit was obtained if we used a log transformation, which means that the marginal effect of diffusion reduces in later time periods. For the choice model, we used the parameters presented in Table 1 and the aggregate attribute values for each year presented in Table 2. As it was not possible to estimate the parameter $M$, we again used 877,000 as the potential market. Then we obtained the parameters for the before and the after data shown in Table 5.

Table 5: Parameter estimates of the diffusion choice model

\begin{tabular}{|c|c|c|c|c|c|c|}
\hline \multirow{3}{*}{ Parameter } & \multicolumn{3}{|c|}{ Before } & \multicolumn{3}{|c|}{ After } \\
\hline & \multicolumn{3}{|c|}{$95 \%$ confidence interval } & \multicolumn{3}{|c|}{$95 \%$ confidence interval } \\
\hline & Value & Min & Max & Value & Min & Max \\
\hline ASC & -12.4906 & -17.996 & -6.9852 & -18.3681 & -20.977 & -15.759 \\
\hline q_ev & 1.7055 & 0.8363 & 2.5747 & 1.8401 & 1.0606 & 2.6197 \\
\hline lambda & 2.6013 & 0.8426 & 4.3601 & 1.5608 & 0.5584 & 2.5632 \\
\hline F value & & 120.91 & & & 123.01 & \\
\hline $\operatorname{Pr}>F$ & & $<.0001$ & & & $<.0001$ & \\
\hline
\end{tabular}

For this model, all the estimated parameters were significant at the $95 \%$ level (i.e. the $95 \%$ confidence interval does not include zero) but with some large confidence intervals. As already seen in the previous Bass model, the diffusion effect is difficult to estimate, as the market is still very small. Simulating with the above parameters allowed us to find that the market is - of course - dependent on the diffusion parameter. We then performed a sensitivity test. If we set the diffusion parameter to the lowest value in the confidence interval, we obtain $1.6 \%$ and $3 \%$, respectively, for the before and after parameters. If, instead, we increase the diffusion effect to be between the minimum and the estimated 
value (we computed it as $90 \%$ from the minimum), we obtained $48 \%$ and $51 \%$ respectively. Although results are sensitive to the diffusion parameter, this example and the results in Figure 3, clearly show that taking into account the effect of diffusion allows predicting a more realistic EV penetration over time.

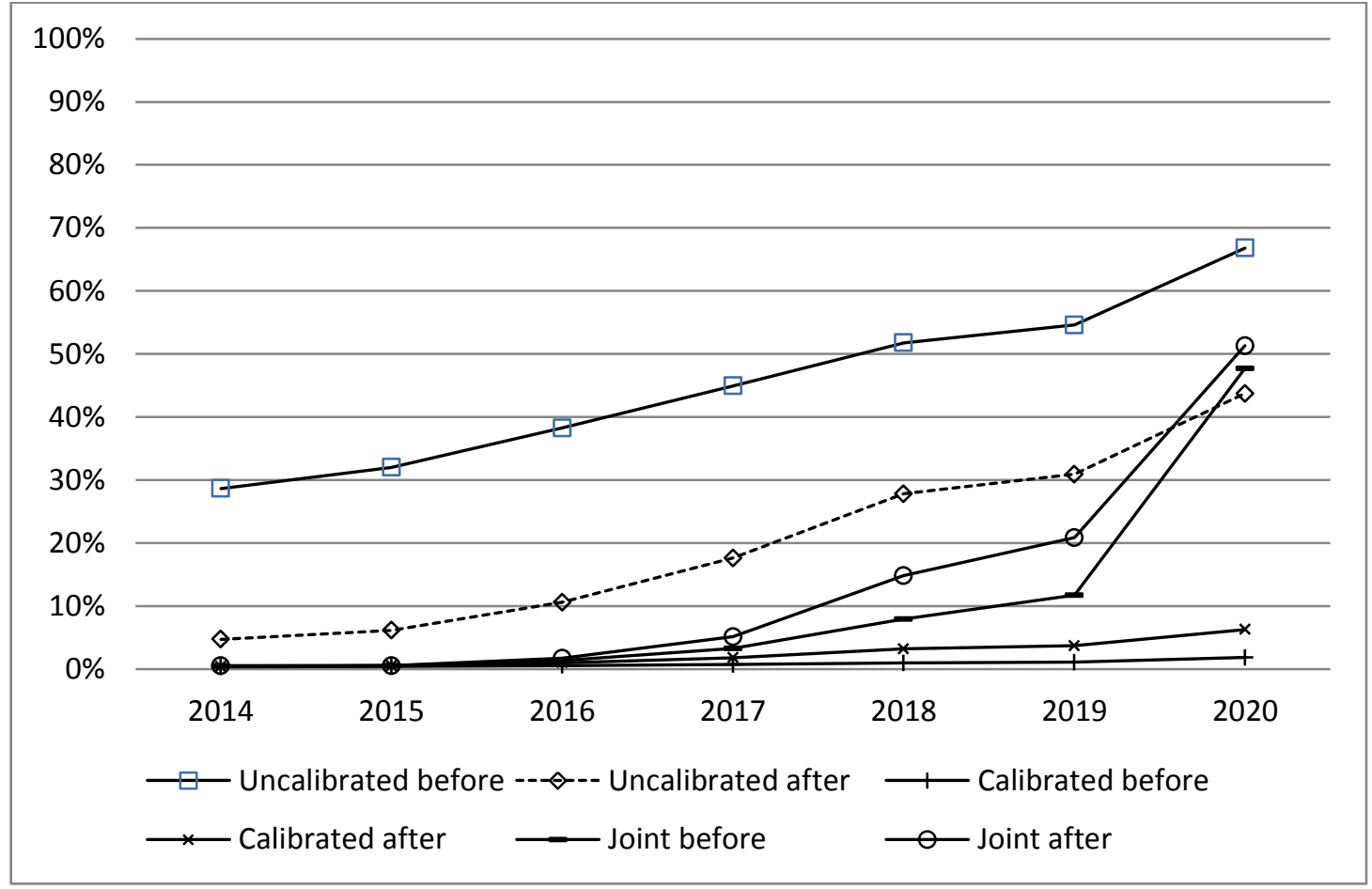

Figure 3: Predicted market shares for the different methods

Figure 3 shows the prediction results obtained (1) using the DCM without (Uncalibrated) and with (Calibrated) recalibrating the ASC to match the base year market shares; and (2) using the joint substitution/diffusion model (Joint). Results from the Joint models illustrate the very low market share at the initial stage and the later (and quick) increase of market share once the product becomes more familiar and available to the population. At the same time, the method proposed in this paper (i.e. integrating the diffusion effect with a more detailed substitution model) allows also to take into account the effect of improving the EV characteristics. This is highly relevant for the EV market as technology is still under development. 
Previous studies have used different versions of growth curve models to describe the future market of EV and similar innovation products. For example Higgins et al. (2012) expected the Australian EV market to remain very low until 2022 followed by a quick increase - to about $10 \%$ - in 2030. But care must be taken when estimating or defining the saturation market limit. For example, using the simpler version of the Bass model, Lamberson (2008) estimated a saturated market of 1.6 million hybrid electric vehicles in the USA in 2007, but from January 2010 to June 2015 more than 2 million hybrid electric vehicles were sold in the USA.

\section{DISCUSSION AND CONCLUSIONS}

This paper proposes a method to forecast EV demand using stated preference data and accounting for the diffusion effect of a new product. Using choice models to simulate the EV market share is problematic, because the current market share used to recalibrate the model in the base year is very low. This means that even with major improvements of the EV attributes, a low market share is obtained in prediction. One of the reasons for this problem is that classic choice models do not account for the effect of diffusion, which is particularly relevant in the case of new products, such as EV.

We suggest a method which combines choice models estimated at the disaggregate level with diffusion models to take into account, jointly, that new products often need time to obtain a significant market share, and that their demand is also strongly affected by the characteristics of the new product. The suggested method allows the improvements on EV attributes not to be overshadowed by the effect of alternative specific constants that constrain the model to the low demand in the early market. Compared to previous studies that combine diffusion models and choice models, our approach allows to account for a much richer formulation of the choice decision (i.e. with several attributes) and suggests a method to re-calibrate the typical alternative specific constants of discrete choice models by explicitly linking them with the diffusion effects. Furthermore, we discuss the effect of direct experience in forecasting and apply our analysis specifically to the market of electric vehicles. 
The method suggested was tested in a very simple case as the data currently available does not provide the foundations for more detailed analyses. The focus has been on showing the strengths of the method suggested rather than providing exact forecasts of the EV market in some specific country. Our results show that taking into account the effect of diffusion allows predicting a more realistic EV penetration over time. It allows explaining the very low market share at the initial stage and a later (and quick) increase of market share once the product becomes more familiar and available to the population. It is important to highlight that the method proposed still requires several assumptions, especially regarding the definition of scenarios, to produce real forecasts. In this paper we define simple scenarios with the objective to test the method proposed, but we do not deal with the problem of defining scenarios.

An important further step in this research could be to focus more on policy tests and also in validating the estimated diffusion/substitution curves. However, this requires some major work because detailed information on real policies is needed (not good assumptions as in our case). It would also require some established market (like for example the Norwegian market) to be used as reference for validation. However, in doing that, we would need to assume that preferences for EV characteristics are the same in Denmark and Norway, and to know the EV characteristics in the Norwegian market over the past years. The first point refers to model transferability, which requires an updating procedure to modify the coefficients so that they represent behaviour in the application context (see Ortúzar \& Willumsen, 2001, page 341). Regarding the second point, we do not know the characteristics of the EV vehicles in Norway over the years; therefore some simplifying assumptions would be needed that would reduce the quality of the validation. Finally, the market share in Norway has been also influenced by strong incentives, the effects of which should be properly accounted in the demand model. But as the demand model estimated in Denmark does not include these incentives, it cannot be validated using Norwegian market shares.

At the same time, the method proposed in this paper integrates the diffusion effect with a more detailed substitution model. A particular strength of the proposed model is that it allows taking into 
account the effects of improving EV characteristics as well as the introduction or removal of specific economic or political incentives. The extent to which it is possible to account for these changes over the years depends on the effects estimated in the substitution model. For example, the stated preference data used in this paper provides specific parameters for EV charging infrastructure. If it is believed (or if we wished to test the effect) that charging infrastructure would be reduced at some point, this can be accounted for in the model and demand would adjust accordingly. If a monetary incentive (e.g. the exemption from registration tax) is believed to last only until a specific year, this can also be accounted for with this model, although our data does not allow to disentangle the pure effect of the tax incentives from that of the purchase price. Other important effects, such as supply strategies and constraints, dealership availability and industrial plans, can also be accounted for if their effect is properly estimated in the demand model. However, to properly account for interactions with the supply system, a dynamic simulation system such as that proposed by Kieckhäfer et al. (2014) would probably be needed.

Finally, our results show that a model estimated after individuals had real-life experience with electric vehicles produces what appear to be more reasonable aggregate market shares, especially for the base year. This result probably depends on our specific data and cannot be generalised, but it is interesting and confirms that results can, of course, be very different if the estimated individual preferences are different from what is typically measured from individuals without experience. The results also suggest that individual preferences will probably change over the year as individuals gain more experience with EV. However, with the data at hand, it is not possible to foresee at what point in time in the future this would happen and how to incorporate this effect properly into the joint discrete/diffusion model. 


\section{Acknowledgements}

This research has been carried out as part of the Green eMotion project. The last author wishes to thank the support of the Institute in Complex Engineering Systems (ICM: P-05-004-F; CONICYT: FBO16) and the Alexander von Humboldt Foundation.

\section{REFERENCES}

Achtnicht, M. (2012). German car buyers' willingness to pay to reduce CO2 emissions. Climatic Change, 113(3-4), 679-697. http://doi.org/10.1007/s10584-011-0362-8

Adler, T., Wargelin, L., Kostyniuk, L., Kalavec, C., \& Occiuzzo, G. (2003). Incentives for alternate fuel vehicles: A large-scale stated preference experiment. In 10th. International Conference on Travel Behaviour Research,. Lucerne.

Bass, F. M. (1969). A New Product Growth for Model Consumer Durables. Management Science, 15(5), 215-227. http://doi.org/10.1287/mnsc.15.5.215

Batley, R. P., Knight, M. J., \& Toner, J. P. (2004). A mixed logit model of U.K. household demand for alternative-fuel vehicles. Rivista Internazionale Di Economia Dei Transporti - International Journal of Transport Economics, 31(1), 55-77.

Beggs, S., Cardell, S., \& Hausman, J. (1981). Assessing the potential demand for electric cars. Journal of Econometrics, 17(1), 1-19. http://doi.org/DOI: 10.1016/0304-4076(81)90056-7

Ben-Akiva, M. E., \& Lerman, S. R. (1985). Discrete choice analysis: theory and application to travel demand (Vol. 9). MIT press.

Brownstone, D., Bunch, D. S., \& Train, K. (2000). Joint mixed logit models of stated and revealed preferences for alternative-fuel vehicles. Transportation Research Part B: Methodological, 34(5), 315-338.

Bunch, D., Bradley, M., Golob, T., Kitamura, R. \& Occhiuzzo, G. P. (1993). Demand for clean-fuel vehicles in California: a discrete-choice stated preference pilot project. Transportation Research, Part A: Policy and Practice, 27(3), 237-253.

Calfee, J. E. (1985). Estimating the demand for electric automobiles using fully disaggregated probabilistic choice analysis. Transportation Research Part B: Methodological, 19(4), 287-301.

Cherchi, E., \& Ortúzar, J. de D. (2006). On fitting mode specific constants in the presence of new options in RP/SP models. Transportation Research Part A: Policy and Practice, 40(1), 1-18.

Christensen, L., Kveiborg, O., \& Mabit, S. L. (2010). The Market for electric vehicles - what do potential users want. In 12th World Conference on Transportation Research. 
Dagsvik, J. K., Wennemo, T., Wetterwald, D. G., \& Aaberge, R. (2002). Potential demand for alternative fuel vehicles. Transportation Research Part B: Methodological, 36(4), 361-384.

Daziano, R. A., \& Bolduc, D. (2013). Incorporating pro-environmental preferences towards green automobile technologies through a Bayesian hybrid choice model. Transportmetrica A: Transport Science, 9(1), 74-106.

Eppstein, M. J., Grover, D. K., Marshall, J. S., \& Rizzo, D. M. (2011). An agent-based model to study market penetration of plug-in hybrid electric vehicles. Energy Policy, 39(6), 3789-3802. http://doi.org/10.1016/j.enpol.2011.04.007

Ewing, G. O., \& Sarigöllü, E. (2000). Assessing Consumer Preferences for Clean-Fuel Vehicles: A Discrete Choice Experiment. Journal of Public Policy \& Marketing, 19(1), 106-118.

Glerum, A., Stankovikj, L., Thémans, M., \& Bierlaire, M. (2013). Forecasting the Demand for Electric Vehicles: Accounting for Attitudes and Perceptions. Transportation Science, 48(4), 483-499. http://doi.org/10.1287/trsc.2013.0487

Goldberg, P. K. (1998). The effects of the corporate average fuel efficiency standards in the US. The Journal of Industrial Economics, 46(1), 1-33.

Gompertz, B. (1825). On the Nature of the Function Expressive of the Law of Human Mortality, and on a New Mode of Determining the Value of Life Contingencies. Philosophical Transactions of the Royal Society of London, 115, pp. 513-583.

Greaves, S., Backman, H., \& Ellison, A. B. (2014). An empirical assessment of the feasibility of battery electric vehicles for day-to-day driving. Transportation Research Part A: Policy and Practice, 66(0), 226-237. http://doi.org/http://dx.doi.org/10.1016/j.tra.2014.05.011

Gunn, H., Fisher, P., Daly, A. J., \& Pol, H. (1982). Synthetic samples as a basis for enumerating disaggregate models. In Proceedings of the 10th PTRC Summer Annual Meeting,. University of Warwick, England.

Gärling, A., \& Thøgersen, J. (2001). Marketing of electric vehicles. Business Strategy and the Environment, 10(1), 53-65. http://doi.org/10.1002/1099-0836(200101/02)10:1<53::AIDBSE270>3.0.CO;2-E

Hackbarth, A., \& Madlener, R. (2013). Consumer preferences for alternative fuel vehicles: A discrete choice analysis. Transportation Research Part D: Transport and Environment, 25(0), 5-17. http://doi.org/http://dx.doi.org/10.1016/j.trd.2013.07.002

Hensher, D. A., \& Greene, W. H. (2001). Choosing between Conventional, Electric and LPG/CNG Vehicles in Single-vehicle Households. In D. A. Hensher (Ed.), The Leading Edge of Travel Behaviour Research, chapter 42 (Vol. 1, p. 725). Oxford: Pergamon Press.

Hidrue, M. K., Parsons, G. R., Kempton, W., \& Gardner, M. P. (2011). Willingness to pay for electric vehicles and their attributes. Resource and Energy Economics, 33(3), 686-705. http://doi.org/DOI: 10.1016/j.reseneeco.2011.02.002

Higgins, A., Paevere, P., Gardner, J., \& Quezada, G. (2012). Combining choice modelling and multicriteria analysis for technology diffusion: An application to the uptake of electric vehicles. 
Technological Forecasting and Social Change, 79(8), 1399-1412. http://doi.org/10.1016/j.techfore.2012.04.008

Ito, N., Takeuchi, K., \& Managi, S. (2013). Willingness-to-pay for infrastructure investments for alternative fuel vehicles. Transportation Research Part D: Transport and Environment, 18(1), 18. http://doi.org/10.1016/j.trd.2012.08.004

Jensen, A. F., \& Cherchi, E. (2014). Exploring different sources of variation in individual preferences for EVs. Paper submitted as a part of $\mathrm{PhD}$ thesis.

Jensen, A. F., Cherchi, E., \& Mabit, S. L. (2013). On the stability of preferences and attitudes before and after experiencing an electric vehicle. Transportation Research Part D: Transport and Environment, 25, 24-32. http://doi.org/http://dx.doi.org/10.1016/j.trd.2013.07.006

Jensen, A. F., Cherchi, E., \& Ortúzar, J. de D. (2014). A long panel survey to elicit variation in preferences and attitudes in the choice of electric vehicles. Transportation, 41(5), 973-993.

Jiang, Z., \& Jain, D. C. (2012). A Generalized Norton-Bass Model for Multigeneration Diffusion. Management Science, 58(10), 1887-1897.

Jong, G. De. (1996). A disaggregate model system of vehicle holding duration, type choice and use. Transportation Research Part B: Methodological, 30(4), 263-276.

Jun, D. Bin, \& Kim, J. il. (2011). A choice-based multi-product diffusion model incorporating replacement demand. Technological Forecasting and Social Change, 78(4), 674-689. http://doi.org/http://dx.doi.org/10.1016/j.techfore.2010.10.012

Jun, D. Bin, \& Park, Y. S. (1999). A choice-based diffusion model for multiple generations of products. Technological Forecasting and Social Change, 61(1), 45-58.

Jun, D. B., Kim, S. K., Park, Y. S., Park, M. H., \& Wilson, A. R. (2002). Forecasting telecommunication service subscribers in substitutive and competitive environments. International Journal of Forecasting, 18(4), 561-581.

Keith, D. R. (2012). Essays on the dynamics of alternative fuel vehicle adoption: insights from the market for hybrid-electric vehicles in the United States. Massachusetts Institute of Technology.

Kerin, R. A., Hartley, S. W., Rudelius, W. R., \& Berkowitz, E. N. (2003). Marketing (Vol. 7). McGraw-Hill/Irwin.

Kieckhäfer, K., Volling, T., \& Spengler, T. S. (2014). A Hybrid Simulation Approach for Estimating the Market Share Evolution of Electric Vehicles. Transportation Science, 48(4), 651-670. http://doi.org/10.1287/trsc.2014.0526

Knockaert, J. (2005). The choice for alternative cars. Energy, Transport and Environment Center For Economic Studies, Leuven, Belgium.

Lamberson, P. J. (2008). The diffusion of hybrid electric vehicles. Future Research Directions in Sustainable Mobility and Accessibility.[Online]. Available from: Http://www. Umsmart. org/project_research/Future_directions. pdf.[9th February 2013]. 
Lilien, G. L., Rangaswamy, A., \& Van den Bulte, C. (2000). Diffusion Models: Managerial Applications and Software. In M. Vijay, E. Muller, \& Y. Wind (Eds.), New-product diffusion models. Springer, Chapter 12.

Mabit, S. L., \& Fosgerau, M. (2011). Demand for alternative-fuel vehicles when registration taxes are high. Transportation Research Part D, 16(3), 225-231.

Ortúzar, J. de D., \& Willumsen, L. G. (2011). Modelling transport (Vol. 4). Wiley.

Pearre, N. S., Kempton, W., Guensler, R. L., \& Elango, V. V. (2011). Electric vehicles: How much range is required for a day's driving? Transportation Research Part C: Emerging Technologies, 19(6), 1171-1184. http://doi.org/10.1016/j.trc.2010.12.010

Potoglou, D., \& Kanaroglou, P. S. (2007). Household demand and willingness to pay for clean vehicles. Transportation Research Part D: Transport and Environment, 12(4), 264-274.

Ramjerdi, F., \& Rand, L. (2000). Demand for Clean Fuel Car in Norway. In Urban Transport Systems. Proceedings from the 2nd KFB Research Conference in Lund, Sweden (pp. 59-78).

Rogers, E. M. (2010). Diffusion of innovations. Simon and Schuster.

Shepherd, S., Bonsall, P., \& Harrison, G. (2012). Factors affecting future demand for electric vehicles: A model based study. Transport Policy, 20, 62-74. http://doi.org/10.1016/j.tranpol.2011.12.006

Struben, J., \& Sterman, J. (2008). Transition challenges for alternative fuel vehicle and transportation systems. Environment and Planning B: Planning and Design, 35(6), 1070-1097.

Train, K. (2009). Discrete Choice Methods with Simulation (Vol. Second Edi). Cambridge University press.

Weerahandi, S., \& Dalal, S. R. (1992). A Choice-Based Approach to the Diffusion of a Service: Forecasting Fax Penetration by Market Segments. Marketing Science, 11(1), 39-53. http://doi.org/10.1287/mksc.11.1.39 